\section{Wenn Arzneimittel zum Allergen werden}

\author{
Unterschiedlichste Arzneimittel können zu Überempfindlichkeits- \\ reaktionen führen. An welche Auslöser man denken sollte und \\ wie man sie sicher identifiziert, zeigen Fallbeispiele, die auf dem \\ 18. Mainzer Allergie-Workshop vorgestellt wurden.
}

Drof rof. Dr. Bernhard Przybilla, München, stellte das Syndrom einer multiplen Arzneimittelallergie war und warnte gleich zu Beginn: „Die Diagnostik von Überempfindlichkeitsreaktionen auf Arzneimittel gehört zu den aufwendigsten und häufig auch frustrierenden allergologischen Aufgaben.“

\section{Patientin mit multiplen Arznei- mittelreaktionen}

Eine heute 37-jährige Patientin erlitt bei einem Sportunfall eine Wirbelsäulenfraktur mit einem inkompletten Querschnittssyndrom. Im Lauf der Behandlung bekam sie 18 verschiedene Medikamente. Sie entwickelte insgesamt dreimal ein makulöses Arzneiexanthem sowie ein lokalisiertes Ekzem an der Injektionsstelle eines Heparin-Präparates.

Pricktestungen ergaben bei Spätablesung nach 24 Stunden positive Reaktionen auf Metamizol und Omeprazol sowie nach 48 Stunden auf Enoxaparin-Natrium. Im Epikutantest zeigten sich Spättypreaktionen auf Carbamazepin, Metamizol, Omeprazol und Pantoprazol sowie als Nebenbefund auf Nickel und Perubalsam. Ein Lymphozytentransformationstest ergab für Carbamazepin einen stark erhöhten Stimulationsindex von 25,8 und für Omeprazol von 6,4. Da die Testergebnisse mit der klinischen Anamnese übereinstimmten, konnte auf Provokationstests verzichtet werden. Abschließend wurde die Diagnose einer multiplen Arzneimittelallergie auf Carbamazepin, Omeprazol, Pantoprazol, Metamizol und EnoxaparinNatrium gestellt.

Wie Literaturrecherchen ergaben, ist dies ein ungewöhnliches Ergebnis. „Unsere Patientin reagierte auf Arzneimittel aus vier unterschiedlichen Substanzklassen“, erklärte Przybilla. Bislang wurde das Syndrom der multiplen Arzneimittelall- ergie nahezu ausschließlich für Antibiotika beschrieben. Der Mechanismus, der den Reaktionen zugrunde liegt, ist noch nicht geklärt. Möglicherweise neigen betroffene Patienten dazu, niedermolekulare Substanzen besonders effektiv als Haptene zu binden. Oder die Gabe verschiedener Fremdstoffe in hohen Dosen führt zu einer Störung der immunologischen Toleranz. „Für die Praxis zeigt das Beispiel unserer Patientin, dass man sich nicht mit einem positiven Testergebnis zufriedengeben, sondern alle fraglichen Medikamente testen sollte“, betonte Przybilla.

\section{CAST hilfreich für die Diagnostik}

Die diagnostische Abklärung von Narkosezwischenfällen ist oft ebenfalls schwierig, erklärte Dr. Britta Opper, Jena. Denn bei Muskelrelaxanzien sind Hauttestreaktionen aufgrund möglicher unspezifischer Histaminliberation unzuverlässig. Provokationstests mit Anästhetika sind nicht möglich. Hilfreich ist ein zusätzlicher zellulärer Antigenstimulationstest (CAST), wie die Kasuistik einer schweren anaphylaktischen Reaktion auf eine Allgemeinnarkose zeigt.

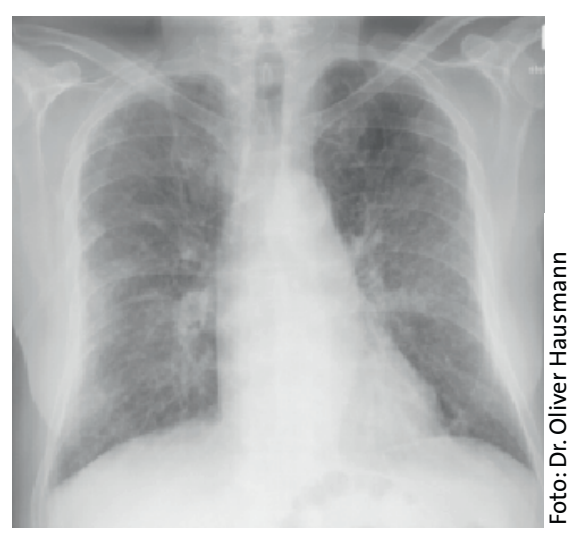

Diffuse beidseitige feinnoduläre Infiltration der Lunge, ausgelöst durch eine antihormonelle Therapie
Die allergologische Diagnostik umfasste neben den verabreichten Medikamenten sowie Naturlatex auch drei weitere Muskelrelaxanzien, für die Kreuzreaktionen bekannt sind. „Wir identifizierten Atracurium als auslösendes Agens, das sowohl im Prick-als auch im Intrakutantest - hier bereits in einer Verdünnung von 1:10.000 - und im CAST zu positiven Reaktionen führte“, sagte Opper. Um falsch positive Ergebnisse auszuschließen, sollten Zellen von gesunden Probanden im CAST mitgestestet werden.

\section{Endokrine Einflüsse auf das Immunsystem?}

Dass auch eine antihormonelle Therapie das Immunsystem in Aufruhr versetzen kann, zeigt der Fall einer akuten eosinophilen Pneumonie. Dr. Oliver Hausmann aus Bern berichtete über einen 73-jährigen Patienten, der sich mit hohem Fieber, trockenem Husten und zunehmender Dyspnoe in der Notfallambulanz vorstellte. In der Anamnese fanden sich keine Hinweise auf allergische oder asthmatische Beschwerden. Radiologisch zeigte sich eine diffuse beidseitige feinnoduläre Infiltration (Abb.) mit laborchemisch markant erhöhten Entzündungszeichen sowie schwerer Oxygenierungsstörung. Auf eine empirische antibiotische Therapie sprach der Patient nicht an. Innerhalb von drei Tagen nahm die initial leichtgradige Eosinophilie bei deutlich erhöhtem ECPWert bis auf 4,22 G/l (32,5\%) zu. Eine akute eosinophile Pneumonie wurde diagnostiziert und eine systemische Glukokortikoidtherapie zeigte prompte Wirkung. „Als Ursache für dieses dramatische Krankheitsbild postulieren wir eine antihormonelle Therapie, die zehn Tage zuvor zur Behandlung eines neu diagnostizierten nicht metastasierten Prostatakarzinoms initiiert wurde“, erklärte Hausmann. abd

Berking C, Thomas P, Przybilla B. Syndrom der multiplen Arzneimittel-Allergie. Opper B, Schliemann S, Hipler U-C, Elsner P. Der zelluläre Antigenstimulationstest (CAST) in der Diagnostik von Narkosezwischenfällen. Hausmann O, Blatter M, Helbling A, Müller UR. Acute eosinophilic pneumonia during antihormonal treatment for prostate cancer. 18. Mainzer Allergie-Workshop, Mainz, 10.-11. März 2006 